\title{
Effect of Coat Permeability on Melt Velocity of Molten Aluminum Alloy in Expendable Pattern Casting Process ${ }^{* 1}$
}

\author{
Sadatoshi Koroyasu ${ }^{* 2}$ \\ Department of Mechanical and Precision Systems, School of Science and Engineering, Teikyo University, Utsunomiya 320-8551, Japan
}

The effect of the coat permeability on the melt velocity of molten aluminum alloy in the expendable pattern casting (EPC) process was investigated experimentally. For eight kinds of coats, the linear relationship between the inter-coat differential pressure and airflow rate was obtained. Coat permeabilities conforming JIS were determined from the gradient of the linear relationship. Using the eight kinds of coats, aluminum alloy plates were cast by the EPC process for three kinds of expansion ratios of expendable polystyrene (EPS) pattern, in the case of bottom pouring and without reduced pressure. The arrival time of the molten metal was measured and the melt velocity was obtained. The use of high expansion ratios of EPS pattern or high permeability coats led to higher melt velocities. In the high coat permeability region, the melt velocity did not increase so much, even when the coat permeability increased. The effects of the pouring temperature and casting thickness on the melt velocity were also examined. The application of high pouring temperature or large casting thickness, led to higher melt velocity. The experimental values of the melt velocity were compared with the calculated values based on the mold filling model used in the previous study. The experimental values were in relatively good agreement with the calculated values, except for when the coat permeability was high and the casting thickness was small. [doi:10.2320/matertrans.F-M2016821]

(Received April 26, 2016; Accepted June 2, 2016; Published July 8, 2016)

Keywords: expendable pattern casting, aluminum alloy, mold filling, coat permeability

\section{Introduction}

The expendable pattern casting (EPC) process is very attractive, because near net shape castings can be obtained without having to divide and assemble the mold and core, even for complex-shaped casting. Therefore, this process makes it possible to eliminate not only machining steps but also the burr finishing process. Furthermore, because a molding binder is not added to the mold in the EPC process, the environment load can be reduced. Because of the extremely high recycling rate of sand, the cost of packing sand can be also reduced, under the situation where silica sand is replaced with artificial sand. In the EPC process, the molten metal is poured into the cavity produced by the thermal decomposition and liquefaction of the expendable polystyrene (EPS) pattern. The thermal decomposition gas and liquid resin discharge into the dry sand through the coat layer. Therefore, the mold filling mechanism in the EPC process is more complicated than when using a cavity mold ${ }^{1)}$. Because of the discharge of the thermal decomposition gas through the coat layer, it is thought that the effect of the coat permeability on the mold filling is significant. When the coat permeability changes, the thickness of the thermal decomposition gas layer between the melt surface and non-decomposition EPS pattern changes. Therefore, it seems that the coat permeability and melt velocity do not have a simple relationship ${ }^{2-4)}$. In addition, when the pouring temperature and expansion ratio of the EPS pattern change, it seems that the generation rate of the thermal decomposition gas changes and the melt velocity also changes. Especially in the EPC process of aluminum alloys, when the melt velocity is very low, a misrun due to a temperature drop at the melt surface ${ }^{5,6)}$ can easily occur. On the other hand, when the melt velocity is too high, because non-decom-

\footnotetext{
${ }^{* 1}$ This Paper was Originally Published in Japanese in J. JFS 88 (2016) 192-197.

${ }^{* 2}$ Corresponding author, E-mail: koroyasu@mps.teikyo-u.ac.jp
}

posed parts of the pattern become trapped within the molten metal ${ }^{1)}$, internal residue resin defects can easily occur. Therefore, it is important to estimate the melt velocity in the EPC process. However, there are few mold filling analysis systems that can exactly simulate the EPC process ${ }^{7-9}$. Therefore, the accumulated experimental data for the exact mold filling analysis are required.

Recently, a relatively large number of investigations have been conducted on mold filling for the EPC process, such as the research on molten cast iron by Maruyama et al. ${ }^{10,11)}$ However, few studies have been performed on the mold filling mechanism in the EPC process, such as on the quantitative effect of process parameters on the melt velocity ${ }^{2,12)}$. Especially on the melt velocity for wide range of the coat permeability, almost no studies have been reported. In a previous experimental study ${ }^{13)}$, for three coats with different permeabilities, an aluminum alloy plate was cast using the EPC process, and effects of the casting design and reduced pressure in the flask on the melt velocity were investigated. It was found that the melt velocity did not increase in proportion to the coat permeability. However, because there were few conditions on the coat permeability, it is thought that the effect of the coat permeability is not sufficiently understood.

In the present work, for eight coats with further wide range of the coat permeability than a previous report, the coat permeabilities were measured. To clarify the effect of the coat permeability on the melt velocity in the EPC process, an aluminum alloy was cast. Additionally, the effects of the pouring temperature, expansion ratio of the EPS pattern, and casting thickness were examined. The experimental values of the melt velocity were compared with the calculated values based on the mold filling model from a previous study ${ }^{2}$.

\section{Experimental Procedure}

\subsection{Measurement of coat permeability}

Figure 1 shows a schematic of the experimental apparatus 


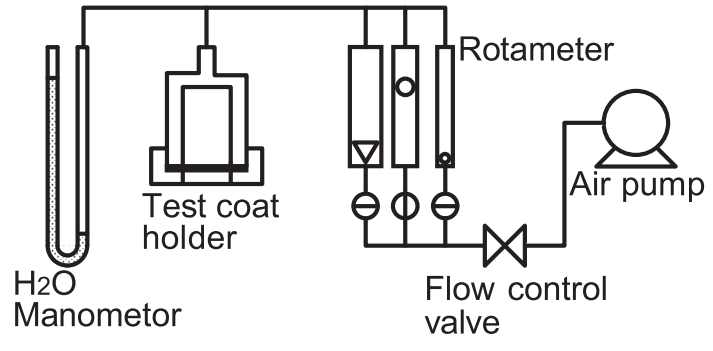

Fig. 1 Schematic diagram of experimental apparatus for coat permeability.

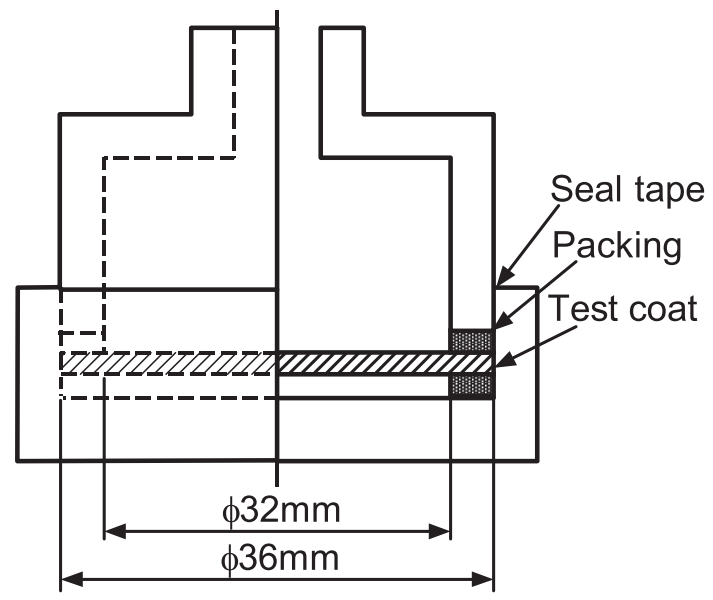

Fig. 2 Schematic diagram of test piece holder for coat permeability.

for measuring the coat permeability. The main body of the apparatus was composed of an air pump, rotameter, test coat layer holder, and $\mathrm{H}_{2} \mathrm{O}$ manometer. Figure 2 shows the details of the test coat layer holder which was composed of a vinyl chloride cylindrical pipe with an inside diameter of $32 \mathrm{~mm}$, O-ring packing, and aluminum alloy ring support of a coat layer with an inside diameter of $36 \mathrm{~mm}$. The coat slurry was poured into an acrylic pattern with an inside diameter of $38 \mathrm{~mm}$ and thickness of approximately $3 \mathrm{~mm}$. After drying, the test coat layer was removed from the pattern. Each test coat layer was formed almost uniform thickness, with a diameter of $35 \mathrm{~mm}$ and thickness of $2-3 \mathrm{~mm}$. The test coat layer was then set between the airtight packing in the test coat layer holder. The air flow rate from the air pump, which passed a test coat layer, was measured by a rotameter. The inter-coat differential pressure was measured by an $\mathrm{H}_{2} \mathrm{O}$ manometer. The maximum differential pressure of the $\mathrm{H}_{2} \mathrm{O}$ manometer was set at approximately $40 \mathrm{cmH}_{2} \mathrm{O}$. This differential pressure was equivalent to an aluminum alloy melt head of approximately $170 \mathrm{mmAl}$. It seems that the condition on this differential pressure was essentially the same as that of the melt head at the melt surface during mold filling, as described in section 2.2. The coat permeability measurements were carried out at room temperature (approximately $293 \mathrm{~K}$ ).

The results of the inter-coat differential pressure were plotted as a function of the air flow rate through the coat layer. Based on the assumption of Darcy flow, the coat permeability was determined from the gradient of the plotting. Therefore, this measurement was in accordance with the method ${ }^{14)}$ based on the JIS mold permeability (JIS Z2603). The coat permea-

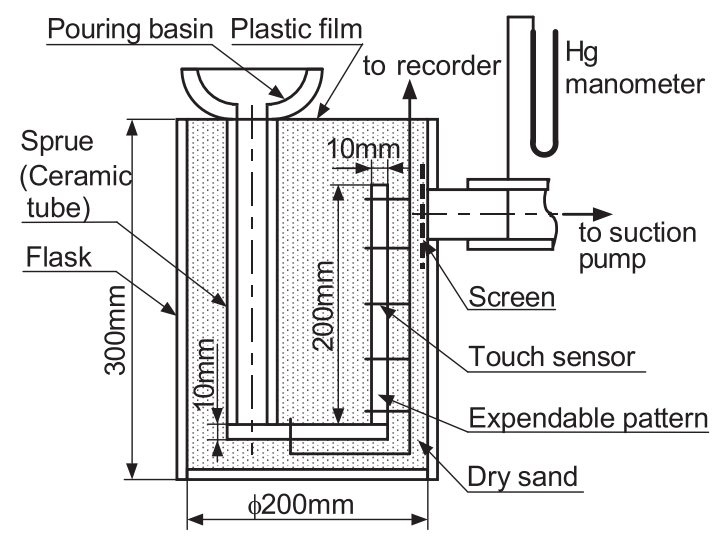

Fig. 3 Schematic diagram of casting apparatus for measurement of mold filling.

bility $K$ is an absolute number with dimension of $\left(\mathrm{cm}^{2}\right.$. $\left.\mathrm{cmH}_{2} \mathrm{O}^{-1} \cdot \mathrm{min}^{-1}\right)$. It is defined in the following equation using the coat thickness $\delta(\mathrm{cm})$, coat layer area $s\left(\mathrm{~cm}^{2}\right)$, volumetric air flow rate $v^{\prime}\left(\mathrm{cm}^{3} \cdot \mathrm{min}^{-1}\right)$, and inter-coat differential pressure $\Delta P\left(\mathrm{cmH}_{2} \mathrm{O}\right)$.

$$
K=\frac{\delta v^{\prime}}{s \Delta P}
$$

A mica based coat (coat A) and seven kinds of silica based coats (coats B-H) were used in this study. The coat layer of mica based coat (coat A) generally has a high insulation characteristics not only a low permeability. The coat permeability of seven kinds of silica based coats was varies by changing the aggregate diameter. Although the thermal conductivity of the coat layer is not mentioned in detail in this paper, the thermal conductivity of the mica based coat (coat A) was approximately one-third that of the silica based coats. Even with different aggregate diameters for silica based coats (coat $\mathrm{B}-\mathrm{H}$ ), the thermal conductivity of the coat layer did not change significantly ${ }^{5)}$.

\subsection{Measurement of melt velocity}

Figure 3 shows a schematic of the casting apparatus used in the experiments, which was similar to that used in a previous study ${ }^{5,6)}$. The steel molding flask was a cylindrical vessel with an inside diameter of $200 \mathrm{~mm}$ and depth of $300 \mathrm{~mm}$. The EPS pattern has a plate shape that is $70 \mathrm{~mm}$ wide, $200 \mathrm{~mm}$ high, and $10 \mathrm{~mm}$ thick. As shown in Fig. 3, the cluster of the bottom pouring system was assembled with the EPS pattern so that the depth direction was $70 \mathrm{~mm}$. The EPS pattern with dimensions of $200 \times 70 \times 5 \mathrm{~mm}$ was used in some cases. Three kinds of EPS patterns with expansion ratios of 30 times $\left(\right.$ density $\left.=29 \mathrm{~kg} \cdot \mathrm{m}^{-}{ }^{3}\right), 60$ times $\left(\right.$ density $\left.=18 \mathrm{~kg} \cdot \mathrm{m}^{-}{ }^{3}\right)$, and 100 times $\left(\right.$ density $=11 \mathrm{~kg} \cdot \mathrm{m}^{-}{ }^{3}$ ) were used. A ceramic tube ( $25 \mathrm{~mm}$ inside diameter, $35 \mathrm{~mm}$ outside diameter, and $300 \mathrm{~mm}$ length) was used as a sprue from the pouring basin to the runner. The pouring basin was an insulating sand mold with an inner diameter of $80 \mathrm{~mm}$, depth of $80 \mathrm{~mm}$, and thickness of $15 \mathrm{~mm}$. The runner was an EPS plate with a cross section of $35 \times 10 \mathrm{~mm}$ and length of $125 \mathrm{~mm}$. The cross section of the ingate was $35 \times 10 \mathrm{~mm}$. Silica sand with a mode diameter of approximately $0.15 \mathrm{~mm}$ (AFS grain fineness number 62) was poured into the flask. 
Touch sensors of the molten metal were inserted into the EPS pattern at 10, 55, 100, 145, and $190 \mathrm{~mm}$ from the ingate, as shown in Fig. 4(a), in order to measure the arrival time of the molten metal for the flow direction distance $y^{11)}$. The touch sensors were tungsten wires with diameters of $0.5 \mathrm{~mm}$. They passed through the center of the $70 \mathrm{~mm}$ wide EPS pattern. As shown in Fig. 4(b), the voltage across the resistance increased in a stepwise fashion, every time the molten metal reached a touch sensor. The arrival time $t_{\mathrm{a}}$ was defined from the increasing time in this voltage change chart. In order to determine the arrival time from the ingate, the time when the molten metal reached the ingate, was required. This time was determined by extrapolation from the times when the molten metal reached $y=10 \mathrm{~mm}$ and $y=55 \mathrm{~mm}$.

Eight kinds of coats as shown in Table 1, which have been measured by the method in 2.1 were used in this study. The EPS pattern was coated using a dipping method. The coated pattern was then dried for $24 \mathrm{~h}$ in a drying furnace at $323 \mathrm{~K}$. Each coat was applied so that the thickness of the coat after drying was approximately $1 \mathrm{~mm}$.

The aluminum alloy JIS AC2A (A319 equivalent) was used as a casting material. It was melted in a high frequency electric induction furnace and cast directly from the furnace. The pouring temperature was set at approximately $1073 \mathrm{~K}$. However, some conditions required a pouring temperature of approximately $973 \mathrm{~K}$. During pouring process, the distance from the bottom of the pouring basin to the melt surface was maintained at approximately $50 \mathrm{~mm}$. As a result, the melt head at the melt surface during mold filling decreased from approximately $350 \mathrm{mmAl}$ to $150 \mathrm{mmAl}$. Only atmospheric pressure was applied as the pressure conditions in the flask during the pouring process and mold filling. Only the bottom pouring system was applied as the casting design, as shown in

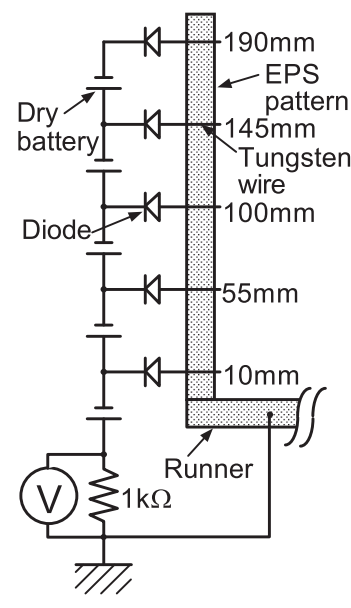

(a)

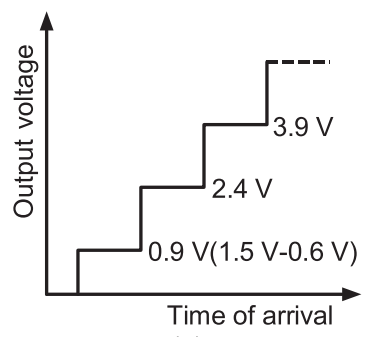

(b)
Fig. 4 Schematic diagram of touch sensor of molten metal. (a) Wiring diagram, (b) Output voltage.

Table 1 Experimental results of coat permeability.

\begin{tabular}{|l|c|c|c|c|c|c|c|c|}
\hline Test cast & A & B & C & D & E & F & G & H \\
\hline$\delta / \mathrm{cm}$ & 0.24 & 0.24 & 0.26 & 0.27 & 0.26 & 0.34 & 0.35 & 0.31 \\
\hline Permeability & 0.13 & 0.41 & 0.81 & 1.7 & 2.6 & 5.1 & 10 & 45 \\
\hline
\end{tabular}

Fig. 3.

\section{Results and Discussion}

\subsection{Experimental results for coat permeability}

Figures 5 and 6 show the experimental results measured by the apparatus for the coat permeability shown in Fig. 1, and show plotting of the inter-coat differential pressure $\Delta P$, as a function of the air flow rate through the coat layer $v$. The parameter $\delta$ in each figure represents the thickness of the test coat layer. Figure 5 shows the experimental results for coats A-C. Figure 6 shows the experimental results for coats D-H. Because the range of the air flow rate $v$ for the differential pressure range $\Delta P=0-40 \mathrm{cmH}_{2} \mathrm{O}$, differs significantly depending on the coat type, the results for coats $\mathrm{A}-\mathrm{C}$ and those for coats D-H were plotted in different figures. For all coats, the figures show a linear relationship passing through the origin, between the differential pressure and air flow rate. Therefore, under the conditions in this work, the air flow rate

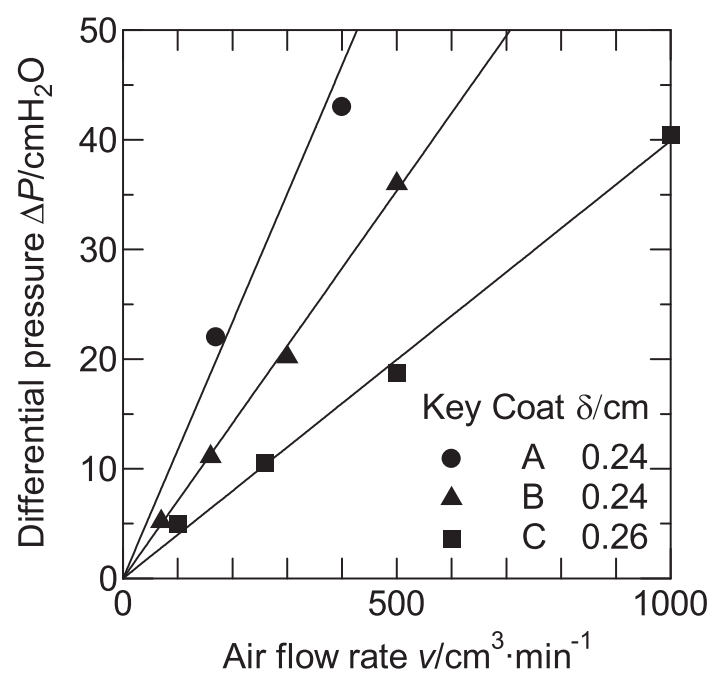

Fig. 5 Relationship between differential pressure $\Delta P$ and air flow rate $v$ for coats A,B and C.

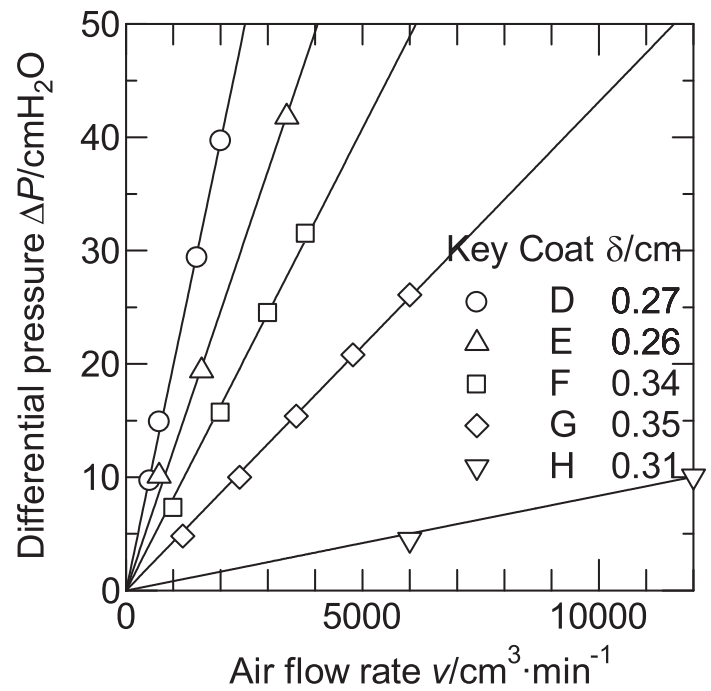

Fig. 6 Relationship between differential pressure $\Delta P$ and air flow rate $v$ for coats D,E,F,G and $\mathrm{H}$. 


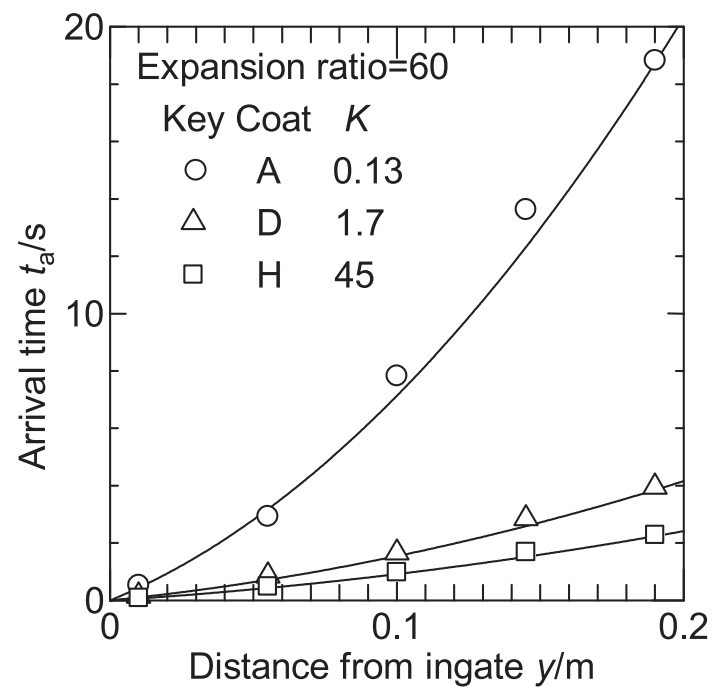

Fig. 7 Effect of coat permeability $K$ on arrival time $t_{\mathrm{a}}$ for pattern expansion ratio of 60 times.

through the coat layer is proportional to the pressure gradient in the coat layer. The air flow in the coat layer is considered to exhibit Darcy flow. As a result, the coat permeability can be calculated by eq. (1). As seen in Figs. 5 and 6, the air flow rate at constant differential pressure increases in the order of coat A to $\mathrm{H}$. The coat permeability also qualitatively increases in this order. In this work, the coat permeability $K$ was determined using the air flow rate $v$ and inter-coat differential pressure $\Delta P$ where the differential pressure is the largest.

Table 1 shows the experimental values of the coat permeability obtained from eq. (1) for coats A-H. The coat permeability of coat A, which was the smallest among the eight kinds of coat shown in Table 1 , is $K=0.13$. Although coat A has high insulating characteristics due to large void as compared with the silica based coats $\mathrm{B}-\mathrm{H}$, the coat permeability is very low due to the air flow which rotates around the mica plate. The differences in the coat permeabilities of coats $\mathrm{B}-\mathrm{H}$ are dependent on differences in the air flow resistance in the aggregate bed due to large differences in the aggregate diameters. The coat permeability of coat $\mathrm{H}$ which has the highest permeability, is $K=45$, which is approximately 350 times that of coat A. The range of test coat thicknesses $\delta$ shown in Figs. 5 and 6 , is $0.24-0.35 \mathrm{~cm}$. In order to examine the effect of test coat thickness, for several kinds of coat layer thickness in the range of $0.2-0.4 \mathrm{~cm}$, the relationships between the inter-coat differential pressure $\Delta P$ and air flow rate $v$ were measured using coat D. It was found that there was little difference in the calculated coat permeability under each condition. Therefore, it is thought that the coat layer thickness had little effect on the measured value of the coat permeability under the conditions in this work.

\subsection{Experimental results for melt velocity}

Figure 7 shows an example of the experimental results of the mold filling for the arrival time of the molten metal $t_{\mathrm{a}}$ as a function of the distance from the ingate at the pattern bottom $y$, for coats $\mathrm{A}, \mathrm{D}$, and $\mathrm{H}$, for a 60 times EPS pattern expansion ratio. The arrival times of the molten metal in Fig. 7 were obtained from the touch censors, as shown in Fig. 4(b). Fig-

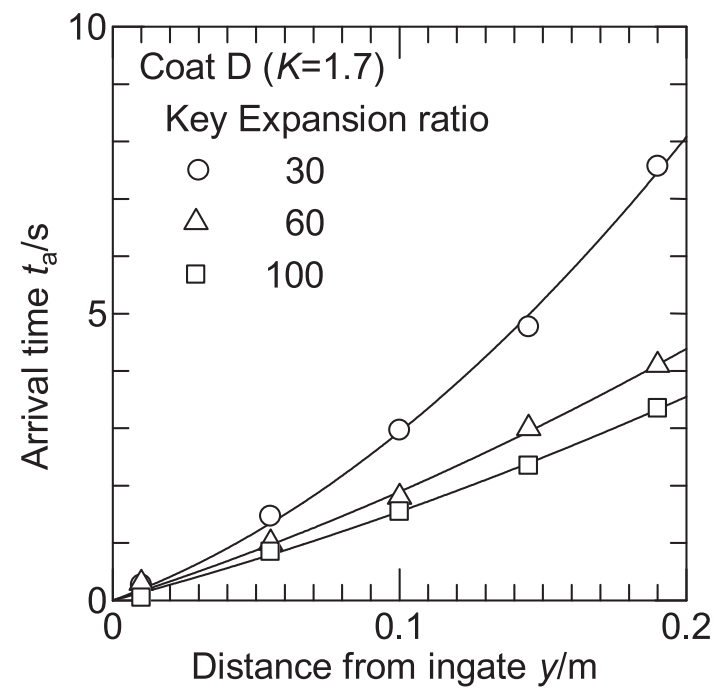

Fig. 8 Effect of pattern expansion ratio on arrival $t_{\mathrm{a}}$ time for coat permeability $K=1.7$.

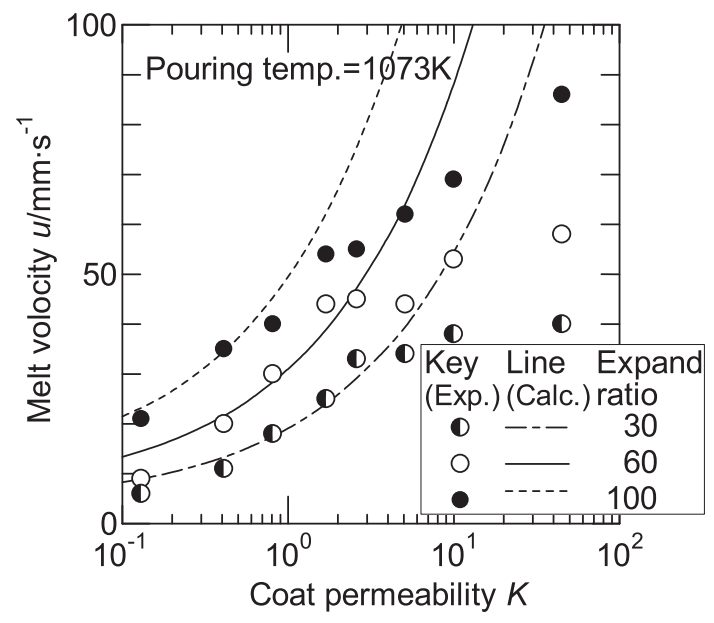

Fig. 9 Effect of coat permeability $K$ on melt velocity $u$ for three kinds of expansion ratios of EPS pattern.

ure 8 shows a plot similar to that in Fig. 7, for the effect of the expansion ratio of the EPS pattern for coat $\mathrm{D}(K=1.7)$. As can be seen in these figures, with increasing the coat permeability $K$ or increasing the expansion ratio of the EPS pattern, the arrival time of the molten metal decreases. As the coat permeability or expansion ratio of the EPS pattern increases, the thickness of the thermal decomposition gas layer decreases. As a result, it seems that the thermal decomposition of the EPS pattern is enhanced by an increase in the heat transfer from the molten metal to the EPS pattern ${ }^{2)}$. As in either case shown in Figs. 7 and 8, with increasing the distance from ingate $y$, the gradient of the arrival time increases, and the melt velocity decreases. The reason for this may be the gradual decrease in the melt head, as the molten metal fills. The tendency described above was also observed under other experimental conditions in this work. Therefore, as the melt velocity in next figures, the average velocity which was calculated from the arrival time difference at $10 \mathrm{~mm}$ and $190 \mathrm{~mm}$, was used.

Figure 9 shows the experimental values of the melt veloci- 
ty $u$, as a function of the coat permeability $K$, with the expansion ratio of the EPS pattern as a parameter. The range of the coat permeability $K$ is 0.13 to 45 , which is a difference of approximately 350 times. Therefore, the horizontal axis is shown on a logarithmic scale. As shown in Fig. 9, with increasing the coat permeability $K$ or expansion ratio of the EPS pattern, the melt velocity increases. As mentioned in Figs. 7 and 8, in the case in which the coat permeability or expansion ratio of the EPS pattern is large, the thickness of the thermal decomposition gas layer is thin. As a result, it seems that the thermal decomposition of the EPS pattern is enhanced by increasing heat transfer from the molten metal to the EPS pattern. When the coat permeability is less than approximately 2 , the melt velocity increases somewhat monotonously with increasing coat permeability. However, when the coat permeability is larger than approximately 2 , there is a decrease in the rate of increase. Although there are some differences according to the expansion ratio of the EPS pattern, the increase in the melt velocity by the change in $K$ from 2.0 to 20 is approximately one-half that in $K$ from 0.2 to 2.0.

The lines in Fig. 9 represent the calculated values based on the mold filling model ${ }^{2}$ from a previous study. A summary of this mold filling model is given as follows. The cases in which the molten metal was poured into the cavity formed by the thermal decomposition of the EPS pattern, and the decomposition gas discharges into the dry sand through the coat layer, were considered. Furthermore, the thermal decomposition and gas discharge were considered to proceed under a dynamic equilibrium state, in which the pressure in the gas layer equal to the melt head was maintained ${ }^{11)}$. Even in the calculated results, because the melt head decreases gradually with increasing the distance from the ingate, the melt velocity also decreases gradually. Therefore, as with the experimental results, the average melt velocity was determined from the arrival time to the distance from the ingate $y=200 \mathrm{~mm}$. When the coat permeability is less than approximately 2 , the experimental values of the melt velocity are in relatively good agreement with the calculated values. On the other hand, when the coat permeability is larger than approximately 2, because the increase in the experimental melt velocity by the increase in the coat permeability decreases, the experimental value of the melt velocity is smaller than the calculated value. The reason for this may be as follows. It is considered that the thermal decomposition rate of the EPS pattern is a finite value, and that the thermal decomposition rate becomes a rate controlling step for the melt velocity. Furthermore, the effects of the resistance of the melt flow at the runner and permeability of the dry sand mold which has a finite value $(\approx 75)^{15)}$, are considered. However, because Fig. 9 does not indicate a clear cause for these results, in the future, a modification of the mold filling model $^{2)}$ in the high permeability region will be necessary. Therefore, it is seemed that accumulated experimental data are required.

Figure 10 shows the experimental values of the melt velocity $u$ in the case of the 60 times expansion ratio of the EPS pattern, as a function of the coat permeability $K$, with the pouring temperature as a parameter. As can be seen in Fig. 10, the use of a high pouring temperature leads to a high melt velocity. When the pouring temperature increases, because of the increase in the melt surface temperature, the heat flux due

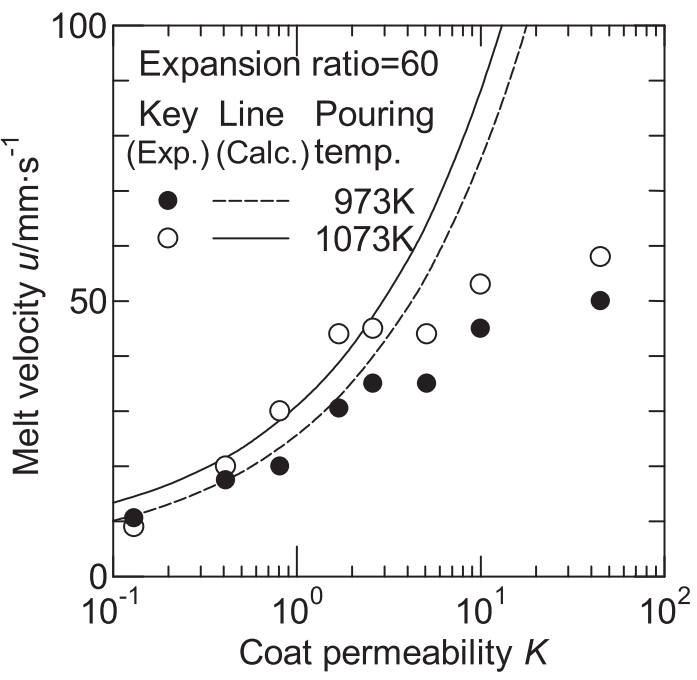

Fig. 10 Effect of pouring temperature on melt velocity $u$ for pattern expansion ratio of 60 times.

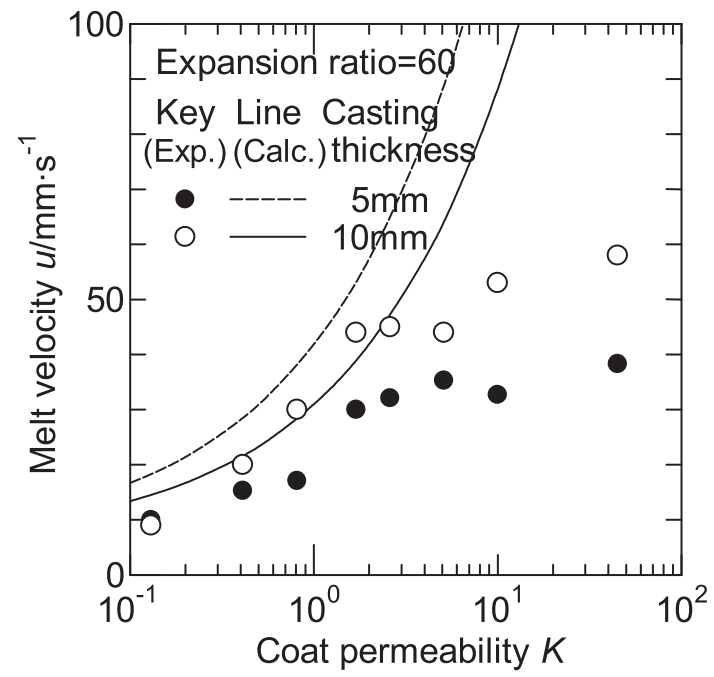

Fig. 11 Effect of casting thickness on melt velocity $u$ for pattern expansion ratio of 60 times.

to the radiation and heat conduction between the melt surface and non-decomposition EPS pattern increases, and the thermal decomposition rate also increases. On the other hand, because of the increase in the thermal decomposition gas thickness, the heat flux by the heat conduction between the melt surface and EPS pattern conversely decreases. Among these effects, it is considered that the former effect is larger. In the calculated values based on the mold filling model shown by each line in Fig. 10, when the pouring temperature is higher, the melt velocity increases by the abovementioned effect. Also, the experimental values of the melt velocity for the pouring temperature of $973 \mathrm{~K}$ were compared with the calculated values. As in the case at $1073 \mathrm{~K}$, when the coat permeability is less than approximately 2 , the experimental and calculated values are in relatively good agreement. When the coat permeability is larger than approximately 2 , the experimental value of the melt velocity is smaller than the calculated value.

Figure 11 shows the experimental values of the melt veloc- 
ity $u$ in the case of the 60 times expansion ratio of the EPS pattern, as a function of the coat permeability $K$, with the casting thickness as a parameter. For the casting thickness of $5 \mathrm{~mm}$, when coat A or coat B which had low coat permeability was used, because the mold filling was incomplete, the average melt velocity was determined from the time to the touch sensor which the molten metal reached finally. As shown in Fig. 11, even in the case of the casting thickness of $5 \mathrm{~mm}$, in the high coat permeability region, the similar tendency in which the melt velocity does not increase so much with increasing coat permeability, is observed. When the casting thickness decreases, the specific surface area of the casting increases, and the generation rate of the thermal decomposition gas per unit coat area decreases. Thus, it is considered that the melt velocity increases by the decrease in the gas layer thickness. Therefore, the calculated value of the melt velocity for the casting thickness of $5 \mathrm{~mm}$ shown in Fig. 11 is larger than that of $10 \mathrm{~mm}$. On the other hand, the experimental values of the melt velocity for the casting thickness of $5 \mathrm{~mm}$ are conversely smaller than those of $10 \mathrm{~mm}$. The reason for this may be as follows. Because the temperature drop of the melt for the casting thickness of $5 \mathrm{~mm}$ is larger than that of $10 \mathrm{~mm}$, the effect of the viscosity increase of the molten metal is significant.

\section{Conclusion}

In order to examine the effect of the coat permeability on the melt velocity of molten aluminum alloy in the EPC process for eight coats with different permeabilities, aluminum alloy plates were cast by the EPC process using three kinds of EPS patterns with different expansion ratios, and the arrival times of the molten metal were measured. The following conclusions were obtained under the conditions of this work.
(1) The use of a high permeability coat or an EPS pattern of high expansion ratio led to a high melt velocity.

(2) As the pouring temperature or casting thickness increased, the melt velocity increased.

(3) In the high coat permeability region, even if the coat permeability increased, the melt velocity did not increase significantly.

(4) Except for the case in which the coat permeability was large and the case in which the casting thickness was small, the experimental values for the melt velocity were in relatively good agreement with the calculated values based on the mold filling model from a previous study.

\section{REFERENCES}

1) F. Sonnenberg: LOST FOAM casting made simple, (American Foundry Society) (2008).

2) S. Koroyasu: J. JFS 81 (2009) 377-383.

3) J. Zhu, I. Ohnaka, T. Ohmichi, K. Mineshita and Y. Yoshioka: J. JFS 72 (2000) 715-719.

4) I Ohnaka, T. Ohmichi, J. Zhu, Y. Hagino, B. Yamamoto and K. Shinano: Report of JFS Meeting 138 (2000) 101.

5) S. Koroyasu and A. Ikenaga: Mater. Trans. 53 (2012) 224-228.

6) S. Koroyasu and M. Matsuda: J. JFS 76 (2004) 687-694.

7) K. Kubo and H. Asao: Report of JFS Meeting 146 (2005) 23.

8) Y. Hotta, H. Yamagata, M. Nikawa, I. Ohnaka, Y. Tate and Y. Mizutani: Report of JFS Meeting 162 (2013) 82.

9) F. Kinoshita: J. JFS 86 (2014) 927-930

10) T. Maruyama, N. Miyazaki and T. Kobayashi: Report of Kansai Branch JFS Meeting (2011) 4-6.

11) T. Maruyama, K. Katsuki and T. Kobayashi: J. JFS 78 (2006) 53-58.

12) M.R. Barone and D.A. Caulk: Int. J. Heat Mass Transfer (2005) 41324149.

13) S. Koroyasu: J. JFS 86 (2014) 447-453.

14) EPC Process Technical Meeting: Characteristic and Standardization of Coat for EPC Process (Kansai Branch of JFS) (1996) 18.

15) S. Koroyasu and M. Matsuda: J. JFS 72 (2000) 85-89. 